\title{
Simulation-based Models of the Galvanic Coupling Intra-body Communication
}

This paper was downloaded from TechRxiv (https://www.techrxiv.org).

\section{LICENSE}

CC BY-SA 4.0

SUBMISSION DATE / POSTED DATE

$13-01-2021 / 13-01-2021$

\section{CITATION}

Ahmed, Doaa; Kirchner, Jens; Fischer, Georg (2021): Simulation-based Models of the Galvanic Coupling Intra-body Communication. TechRxiv. Preprint. https://doi.org/10.36227/techrxiv.13568402.v1

$\mathrm{DOI}$

10.36227/techrxiv.13568402.v1 


\title{
Simulation-based Models of the Galvanic Coupling Intra-body Communication
}

\author{
Doaa Ahmed, Georg Fischer, and Jens Kirchner \\ Lehrstuhl für Technische Elektronik, Friedrich-Alexander-Universität Erlangen-Nürnberg \\ Erlangen, Germany \\ Email: doaa.ahmed, georg.fischer, jens.kirchner@fau.de
}

\begin{abstract}
Human body communication (HBC) uses the human body as a transmission medium for electrical signal at very low power consumption. In this paper, we provided two simulationbased arm models. In the first model, we studied the effect of introducing different types and thicknesses of tissues in the path of signal transmission on the detected signal. In the second model, we introduced different bending angles to the model and examined its effect on the detected signal. The results showed that the signal attenuates very rapidly in the skin tissue. On the other hand, no signal decay is noticed in the fat tissue. Despite the highest attenuation constant of the muscle tissue, adding it the model improves the detected signal at receiver. Increasing the thickness of the muscle layer reduces the electric potential difference at transmitter, while improves the detected signal at receiver. For the bended model, we found that the bending helps to improve the received signal at small angles because the signal takes the shortest path from transmitter to receiver. For larger angles, the discrepancy between this short path and main transmission length will be reduced until they are equal at angle $180^{\circ}$.
\end{abstract}

Index Terms-human body communication, intra-body communication, galvanic coupling, simulation

\section{INTRODUCTION}

Wireless body area communication networks (WBANs) include devices that exchange information in/on the human body or at its proximity. Different techniques for data transmission can be used in WBANs: Radio frequency (RF) propagation, ultrasonic waves, molecular communication, or human body communication (HBC) [1]. RF propagation uses electromagnetic waves for sending data between transceivers using antennas, this technique is appropriate for applications that require high data rates and relatively large transmission range, $10 \mathrm{~m}$ around the body, e.g., sending videos [2]. On the other hand, acoustic waves are used at non-audible frequencies for underwater communications because of the less attenuation transmission compared to RF propagation. Hence, they are now being used for data transmission inside the human body, which is mainly composed of water [1], [3]. In molecular communication, small particles, i.e., chemical/biocompounds, or synthesized nanoparticles, are used as information carriers between transceivers distributed at various length scales that extend from nanometers up to a few meters. This technique is more appropriate for nanomedicine applications because of its bio-compatibility. In this paper, we focus on the galvanic coupling HBC that considers the human body as a transmission medium for electrical signals using electrodes. The signals are confined to the human body with an operating frequency band centered at $21 \mathrm{MHz}$ as stated in the IEEE standard for HBC (IEEE Std 802.15.6-2012) [4]. This frequency band confirms high security, less interference with neighboring transceivers, less signal attenuation through the body, and less radiation [2]. This technique is appropriate for low power consumption applications, e.g., sending vital signs. One application is shown in Fig. 1: Different sensors are distributed across the body of a patient, which collect his vital signs, e.g., ECG, temperature, blood glucose level, etc., then send this data to a central processing unit (CPU) to be combined, encoded and forwarded to an $\mathrm{HBC}$-enabled device that has the ability to decode and translate signals into audible sounds. This will help blind patients to monitor their vital signs individually just by touching the device and take the required action, if possible.

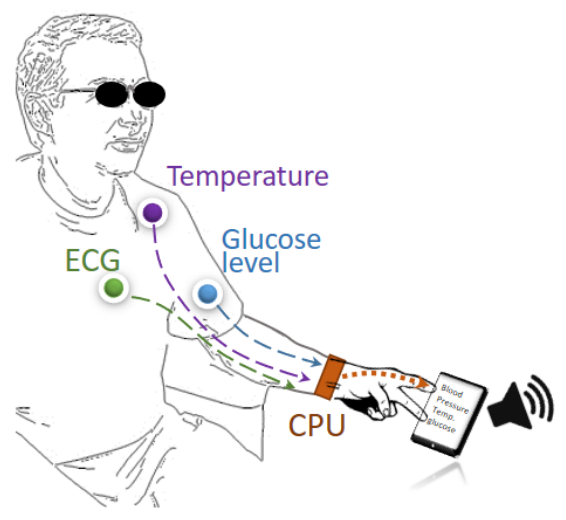

Figure 1: Potential application scenario for intra-body communication: Vital data from several sensors are transmitted to a central hub and processing unit; relevant data are further sent to an HBC-enabled device for displaying.

Several studies were previously done to investigate the transmission mechanism in HBC. In [5], M. Wegmueller et al. compared simulations with measurements to study the influences from the electrode size, human joints between transmitter (TX) and receiver (RX), and electrode positions on signal attenuation in the human body. The mathematical modeling, simulation and system implementation of intra-body communication were reviewed in [6]. M. Callejón et al. [7] simulated the electric field and current density through differ- 
ent tissues as a function of frequency, in addition to studying the influence of different channel lengths on the signal pathloss at an operating frequency of $40 \mathrm{kHz}$. A four terminal circuit model was proposed by K. Ito and Y. Hotta in [8] to describe the signal transmission of the galvanic coupling HBC. In addition, they validated their model using simulations. In [9], we used simulation to investigate the transmission mechanism of signals in intra-body communication and explore the effect of changing the applied frequency, induced current, transmission distance, inter-electrode distances on the detected signal. In [10], we started to investigate the signal transmission in intra-body between sensors distributed on the same body and inter-body communications between sensors distributed on different bodies using simulation.

In this paper, we studied the effect of having different tissues and bending angles between TX and RX on signal transmission. Two different simulation-based models of the human arm were implemented using COMSOL Multiphysics 5.3a. One represents a straight arm model of 7 different geometries, in each geometry we changed the thicknesses of some layers, i.e., introducing more muscles (athletes) or more fats (obese), to investigate how this affects the electric potential difference at transmitter and receiver. This differs from most papers in literature, in which the authors apply a specific potential difference at transmitter and measure the detected signal directly at receiver. In the second model, some bending was applied to the model with angles start from $45^{\circ}$ with an increment of $45^{\circ}$ until $180^{\circ}$. This imitate the movement of the forearm away from the the upper arm.

This paper is organized as follows. In section II, the model geometry and simulation setup are given. The results are collected and discussed in section III. Finally, section IV concludes our work.

\section{Model GeOMetry AND Simulation SETUP}

In galvanic coupling, part of the current, injected through the signal electrode of the transmitter, flows between the transmitting electrodes, while the rest travels along the model for being detected at RX as seen in Fig. 2. In on-body communication the electrodes are placed on the body, which means that the outermost 3 layers, i.e., skin, fat, and muscle, are of more importance and have a large effect on the obtained signals than the bone layers. Therefore, in order to analyze the detected signal from the multilayer geometries, we studied the effect of each tissue separately using geometries $1-3$. In these geometries, a three-layer concentric cylinder of radius $3.5 \mathrm{~cm}$ and length $60 \mathrm{~cm}$ was used. The layers are of thicknesses $2.256,0.6$ and $0.644 \mathrm{~cm}$. The outermost layer was used to represent skin, fat, or muscle in geometries $1-3$, respectively. The second and third layers were used to represent cortical and cancellous bones, respectively. The cross-sectional areas and tissues thicknesses of the proposed geometries are collected in table I. In geometry 4, the human arm was approximated by a five-layer concentric cylinder of radius $3.5 \mathrm{~cm}$ and length $60 \mathrm{~cm}$, see Fig. 3(a) and (b). The layers are skin, fat, muscle, cortical bone and cancellous bone of thicknesses $0.126,0.58$,

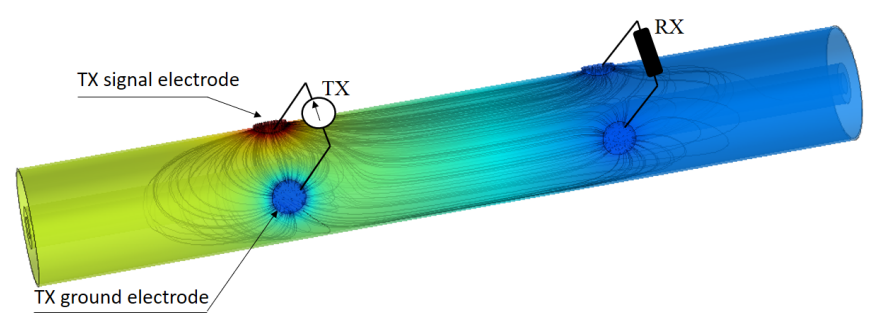

Figure 2: Induced current density inside the arm model.

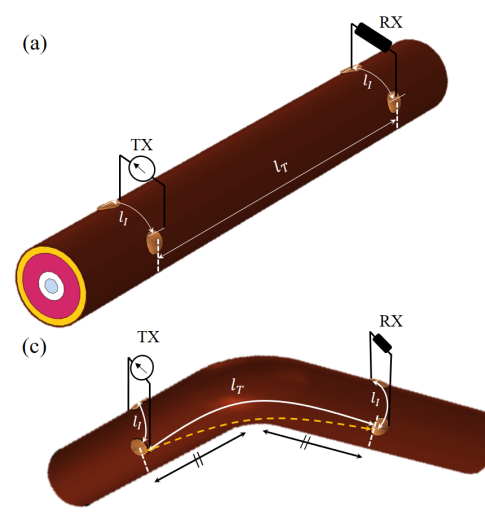

(b)

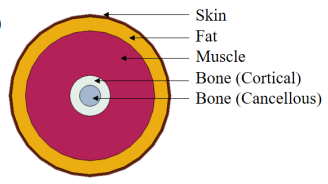

Figure 3: Simulation-based model of a single human arm: (a) 3D straight model, (b) cross sectional view, (c) 3D bended mode, (d) side view of the 3D bended model.

$1.55,0.6$ and $0.644 \mathrm{~cm}$, respectively. These values were chosen to be within the anatomical range [11]-[14]. In the fifth and sixth geometries, we have increased the thickness of the fat layer to be 0.75 and $1.10 \mathrm{~cm}$, respectively; this represents an excess of body fat. However, the increment is taken only from the muscle layer, while the thicknesses of all other layers are kept the same as in geometry 4 . In geometry 7 , the muscle layer was removed and replaced by a fat tissue.

In the second study, we investigated the effect of the arm bending on the received signal based on geometry 4 . Different bending angles, $\theta$, have been applied to the model, starting from $45^{\circ}$ with an increment of $45^{\circ}$ until $180^{\circ}$, i.e., that represents the straight model. This imitates the movement of the forearm towards/away from the upper arm. The transmitting and receiving electrodes were placed symmetrically around the bending as seen in Fig 3(c) and (d).

The dielectric properties of the tissues are represented numerically using a summation of 4-Cole-Cole equations, which describe the complex relative permitivitty of the human tissues as a function of frequency as follows [15]:

$$
\begin{aligned}
\epsilon_{r}^{*}(\omega)= & \epsilon_{\infty}+\sum_{m=1}^{4} \frac{\Delta \epsilon_{m}}{1+\left(j \omega \tau_{m}\right)^{1-\alpha_{m}}}+\frac{\sigma_{i}}{j \omega \epsilon_{0}} \\
= & \epsilon_{r}-j \epsilon_{r}^{\prime \prime}=\epsilon_{r}-j \frac{\sigma}{\omega \epsilon_{0}}
\end{aligned}
$$

where $\epsilon_{r}^{*}(\omega)$ represents the frequency-dependent complex relative permittivity, $\epsilon_{\infty}$ is the material permittivity at very high frequencies, $\Delta \epsilon_{m}, \tau_{m}$, and $\alpha_{m}$ are material parameters for 
Table I: Cross-sectional area and tissue thicknesses in $(\mathrm{cm})$ of proposed geometries used in the simulation

\begin{tabular}{|c|c|c|c|c|c|c|c|}
\hline Geometry & 1 & 2 & 3 & 4 & 5 & 6 & 7 \\
\hline Skin & 2.256 & 0.000 & 0.000 & 0.126 & 0.126 & 0.126 & 0.126 \\
\hline Fat & 0.000 & 2.2564 & 0.000 & 0.580 & 0.750 & 1.100 & 2.130 \\
\hline Muscle & 0.000 & 0.000 & 2.256 & 1.550 & 1.380 & 1.030 & 0.000 \\
\hline Cortical bone & 0.600 & 0.600 & 0.600 & 0.600 & 0.600 & 0.600 & 0.600 \\
\hline Cancellous bone & 0.660 & 0.660 & 0.660 & 0.660 & 0.660 & 0.660 & 0.660 \\
\hline
\end{tabular}

each $m^{\text {th }}$ dispersion region, $\sigma_{i}$ is the ionic conductivity, $j$ denotes the imaginary unit $\sqrt{-1}, \omega$ is the angular frequency, $\epsilon_{0}$ the permittivity of free space, $\epsilon_{r}$ is the relative permitivitty, $\epsilon_{r}^{\prime \prime}$ is the dielectric damping that accounts for losses in the tissue and $\sigma$ is the conductivity. Figure 4 shows the attenuation constant $\alpha$ and dielectric properties of the tissues used in our model in the applied frequency range. However, because the muscle tissue has higher relative permittivity and conductivity than the other tissues, we preferred to use another red (right side) scale that applies only to it. The attenuation constant of the signal travels through the human tissues can be calculated according to [16]:

$$
\alpha=\omega\left\{\frac{\mu_{0} \epsilon_{r} \epsilon_{0}}{2}\left[\sqrt{1+\left(\frac{\epsilon_{r}^{\prime \prime}}{\epsilon_{r}}\right)^{2}}-1\right]\right\}^{1 / 2}(\mathrm{~Np} / \mathrm{m})
$$

where $\mu_{0}$ is the permeability of free space.

Circular electrodes made of copper of thickness $0.2 \mathrm{~cm}$ and radius $1 \mathrm{~cm}$ were used. In galvanic coupling approach, the electrodes should be in direct contact to the body. The transmission distance $l_{T}$ between TX and RX and the interelectrode distance $l_{I}$ were taken $20 \mathrm{~cm}$, and $3.5 \mathrm{~cm}$, respectively. A current controlled harmonic waveform of amplitude $1 \mathrm{~mA}$ was injected from the signal electrode of the transmitter with a frequency sweep from $10 \mathrm{kHz}$ up to $1 \mathrm{MHz}$. The current was chosen to meet the human safety standardization according to [17]. The frequency range was chosen to be above the biological signal frequency and within the range assigned by IEEE 802.15.6 [4], such that quasi-static approximation can be applied and to prevent the body from acting as an antenna at shorter wavelengths. The domain around the model is air that extends to infinity. Extremely fine mesh sizes were applied to all models introduced in this paper. This meshing leads to a number of domain elements between 341782 and 902177, boundary elements between 26130 and 97832, with edge elements between 666 and 1018 .

\section{RESULTS}

The electrical potential difference at TX and RX in addition to the signal attenuation factor for different geometries are
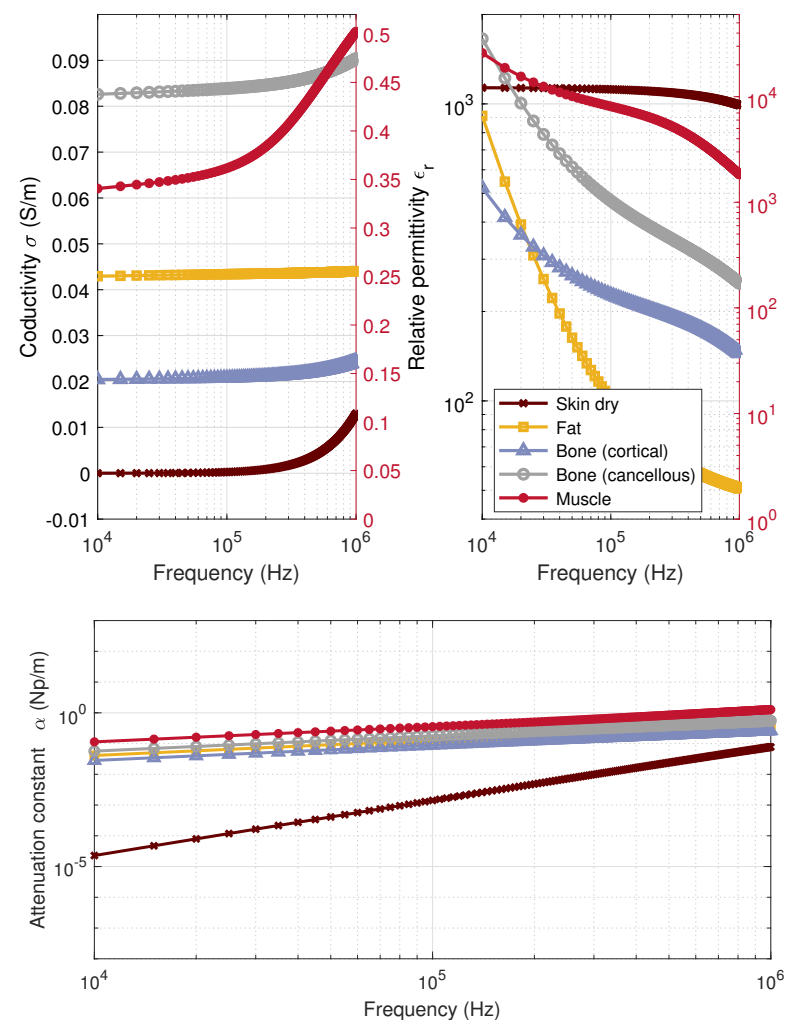

Figure 4: Dielectric properties and attenuation constant of different tissues at different applied frequency (the second red scale applies only to the muscle tissue).

illustrated in Fig. 5. The attenuation factor between TX and RX was calculated according to [5]

$$
\text { Attenuation }[\mathrm{dB}]=20 \cdot \log _{10} \frac{\Delta V_{\mathrm{RX}}}{\Delta V_{\mathrm{TX}}}
$$

where $\Delta V_{\mathrm{RX}}$ and $\Delta V_{\mathrm{TX}}$ denote the potential difference at the receiver and transmitter electrodes, respectively. As expected, changing the geometry changes the detected signals at TX and RX. One reason for that is the impedance of each tissue, which follows the general Ohm's law, in which the resistance 
(a)

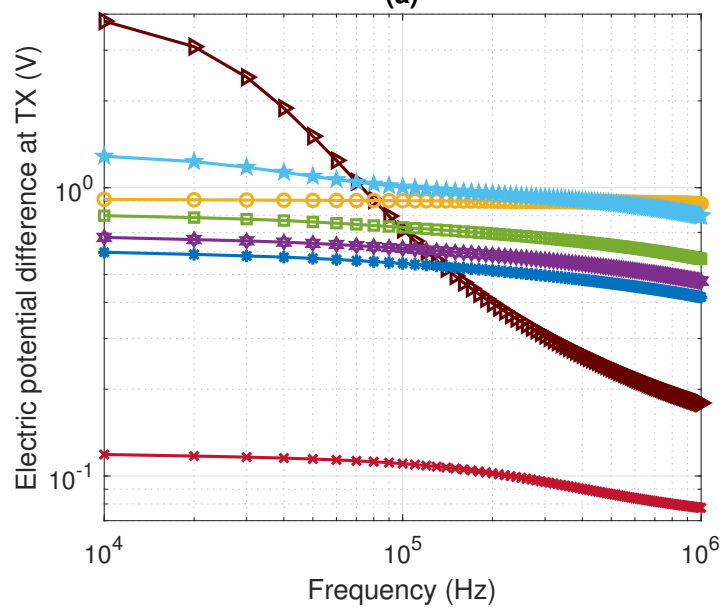

(b)

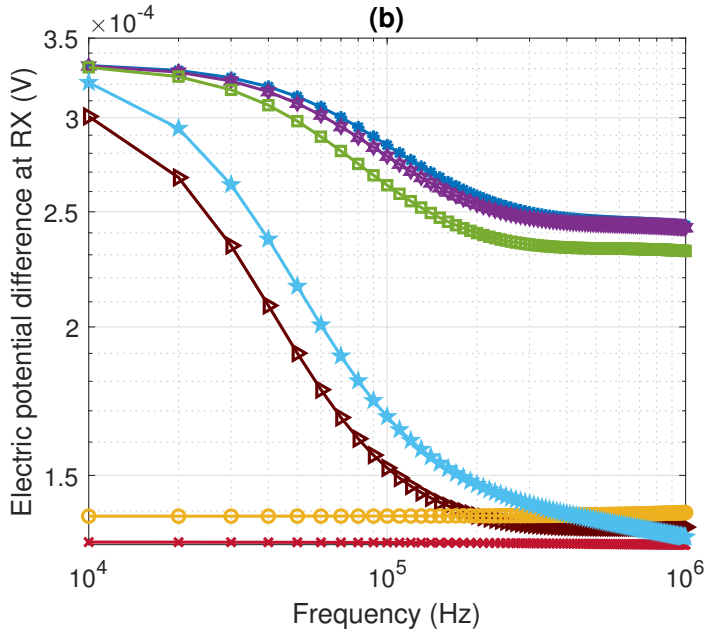

(c)

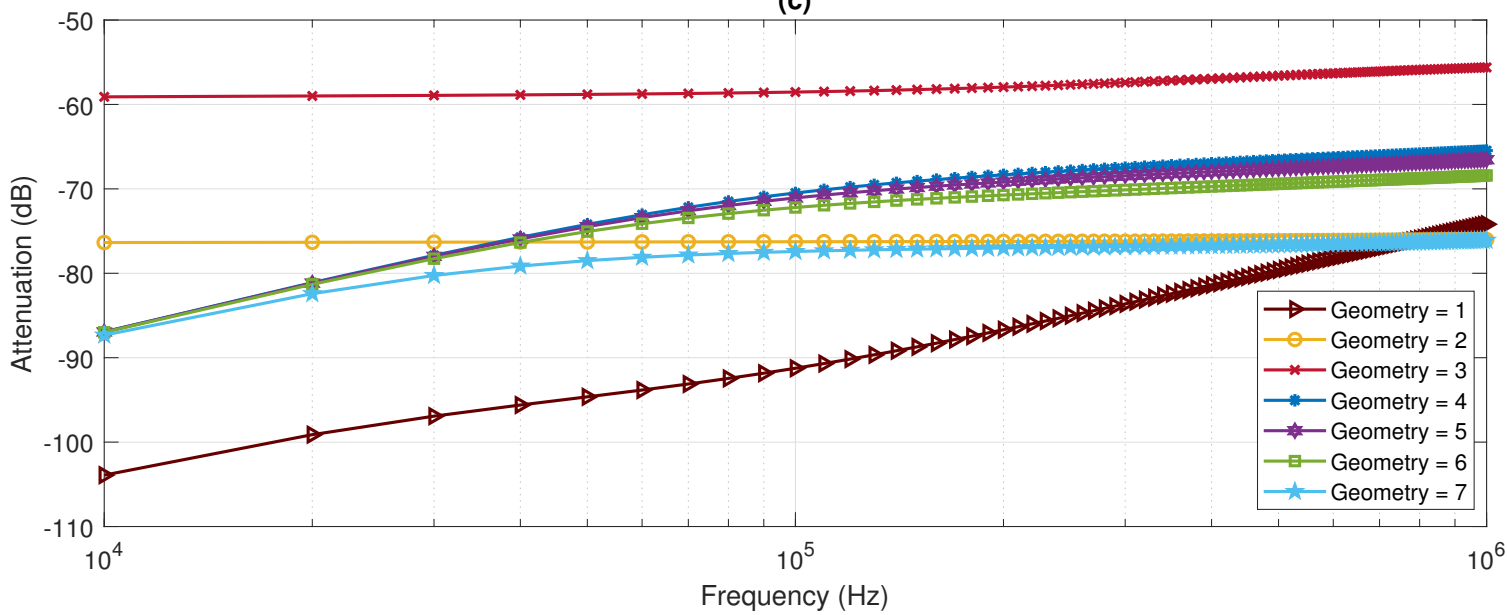

Figure 5: Obtained signals: (a) Electric potential difference at TX, (b) Electric potential difference at RX, (c) Attenuation factor between TX and RX.

is directly proportional to transmission length and inversely proportional to conductivity and surface area. Increasing the resistance will increase the electric potential when current is not changed, as stated in Ohm's law. We started with results from geometries $1-3$ to understand the effect of each tissue separately on the detected signal. Here, the surface area and transmission length are the same for the three geometries, hence only the conductivity of each tissue affects its resistance.

From the dielectric properties of tissues in Fig. 4, we concluded the following:

Skin tissue: the conductivity increases with frequency, which means less detected signal as frequency increases. In addition, the attenuation constant increases very rapidly compared to all other layers over frequency, which explains the quick de- cay of signal over frequency as it travels through the skin in Fig. 5 (geometry 1).

Fat tissue: the conductivity is almost constant, while a small increase in the attenuation constant is noticed in the applied frequency range. This explains the constant behavior of the detected signal over frequency for geometry 2, seen in Fig. 5.

Muscle tissue: it has the highest conductivity and hence attenuation constant over all other tissues, which explains why the detected signal from geometry 3 in Fig. 5 is the weakest. Also, the conductivity increases as the frequency increases. However, the slope of the attenuation constant is small, which explains the small decay of the detected signal over frequency. 


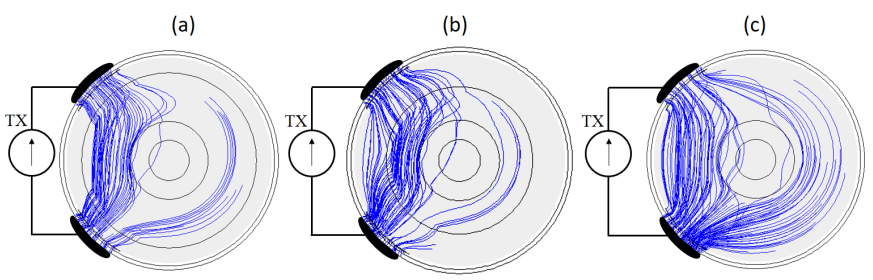

Figure 6: Cross sectional area with the induced current density at TX at frequency $10 \mathrm{kHz}$ : (a) geometry 4, (b) geometry 6 , and (c) geometry 7 .

As seen in Fig. 6, the part of the induced current between TX electrodes flows through subsequent tissues with different dielectric properties, i.e., impedances in series, that contribute to the detected potential difference at TX. Each tissue can be represented by a resistor and capacitor that are in parallel. From geometry 7 in Fig. 5, we found that adding a thin skin layer to a thick fat layer improves the detected signal at RX. On the other hand, at TX, where the signal is injected into the body, the fat layer has a larger contribution to the detected signal at frequencies above $100 \mathrm{kHz}$, where the skin conductivity increases and hence resistivity decreases. Comparing geometry 4 with geometries 5 and 6 , it can be seen that due to the larger thickness of the muscle layer in geometry 4 , it has larger resistance and hence a significant effect on the signal detected at TX, which is lower than that from geometries 5 and 6 with the smaller muscle thicknesses and hence less resistance. In model 5 , we introduced a smaller increase in the fat tissue than that been used in model 4 . Increasing the fat tissue reduces the detected signal at the receiver. However, because a small increase was introduced to the fat thickness, this results in an insignificant decrease in the detected signal at receiver. Increasing the thickness of the muscle layer reduces the potential difference at TX but improves it at RX. This illustrates that the signal attenuation during transmission is affected by the dielectric parameters, thickness of tissues and model geometry.

Fig. 7 shows the electric potential difference at the receiver and the attenuation factor as calculated according to (3). The bending improves the received signal. The reason for that is the actual transmission length. Although we used the same transmission distance $l_{T}=20 \mathrm{~cm}$ for all models with different angles, the actual transmission length, shown as dashed yellow lines in Fig. 3(c) and (d), differs according to the geometry. The signal follows the shortest path between TX and RX, which explains the signal improvement we obtained at the smallest angle $45^{\circ}$. Increasing the bending angle will reduce the discrepancy between the shortest distance between TX and $\mathrm{RX}$ and the transmission length $l_{T}$ until they are equal at angle $\theta=180^{\circ}$.

\section{CONCLUSION}

In human body communication, researchers exploit the dielectric properties of the human tissues to send data through the body rather than air. Galvanic coupling is one approach
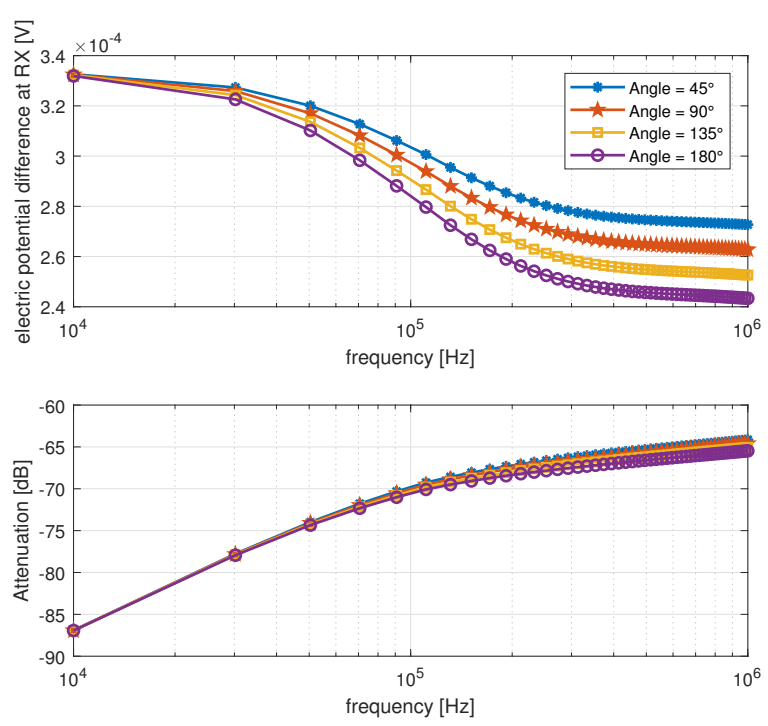

Figure 7: Electric potential difference at RX and the attenuation factor for different angles of the geometry.

used to inject an information carrying electrical signals into the body, in which electrodes should be in direct contact to the human body. In this paper, we built two simulated models of the human arm approximated by a multilayer concentric cylinder. In one model, we investigated the effect of having different types and thicknesses of tissues between TX and RX. In the second model, we studied the effect of having different bending angles, when the transmitting and receiving electrodes are placed symmetrically around the curvature. The results showed that, having a thin fat layer between skin and muscle tissues improves the received signal, which is a good news for athletes. Although the muscle tissue has the highest conductivity and attenuation constant, introducing it to the model improves the received signal. However, having a large electric potential at TX does not indeed mean a large potential difference at RX. The tissue thickness and type has a significant effect on the detected signal and current distribution inside the geometry. Introducing some bending at short distances between TX and RX will improve the detected signal. Increasing the angle of bending will reduce the discrepancy between the shortest distance between TX and $\mathrm{RX}$ and transmission length $l_{T}$ which results in a decrement in the detected signal.

For future work, the authors plan to validate the results from the simulation-based models using experimental measurements.

\section{REFERENCES}

[1] R. Cavallari, F. Martelli, R. Rosini, C. Buratti, and R. Verdone, "A survey on wireless body area networks: Technologies and design challenges," IEEE Communications Surveys Tutorials, vol. 16, pp. 1635-1657, Third 2014.

[2] M. Seyedi, B. Kibret, D. T. H. Lai, and M. Faulkner, "A survey on intrabody communications for body area network applications," IEEE Transactions on Biomedical Engineering, vol. 60, pp. 2067-2079, Aug 2013. 
[3] J. Zhao, X. Chen, B. Liang, , and Q. Chen, "A review on human body communication: Signal propagation model, communication performance, and experimental issues," Wireless Communications and Mobile Computing, Hindawi, vol. 2017, no. 5842310, 2017.

[4] "IEEE Standard for Local and metropolitan area networks - Part 15.6: Wireless Body Area Networks," IEEE Std 802.15.6-2012, pp. 1-271, Feb 2012.

[5] M. Wegmueller, A. Kuhn, J. Froehlich, M. Oberle, N. Felber, N. Kuster, and W. Fichtner, "An attempt to model the human body as a communication channel," IEEE Transactions on Biomedical Engineering, vol. 54, pp. 1851-1857, Oct 2007.

[6] Y. Song, Q. Hao, , and K. Zhang, "Review of the modeling, simulation and implement of intra-body communication," ScienceDirect, Defence Technology, vol. 9, pp. 10-17, Mar 2013.

[7] M. Callejón, J. Reina-Tosina, D. Naranjo-Hernández, and L. M. Roa, "Galvanic coupling transmission in intrabody communication: A finite element approach," IEEE Transactions on Biomedical Engineering, vol. 61, pp. 775-783, March 2014.

[8] K. Ito and Y. Hotta, "Signal path loss simulation of human arm for galvanic coupling intra-body communication using circuit and finite element method models," in 2015 IEEE Twelfth International Symposium on Autonomous Decentralized Systems, pp. 230-235, March 2015.

[9] D. Ahmed, J. Kirchner, and G. Fischer, "Wave propagation with hbc in a human arm model," in 2017 IEEE International Symposium on Medical Measurements and Applications (MeMeA), pp. 448-452, May 2017.

[10] D. Ahmed, J. Kirchner, and G. Fischer, "Signal transmission with intrabody and inter-body communications (simulation-based models)," in 13th EAI International Conference on Body Area Networks (BODYNETS 2018), October 2018.

[11] P. Wilkinson and R. Millington, "Skin (digitally printed version)," in Cambridge University Press, pp. 49-50, 2009.

[12] I. Hwang and K. Shin, "Fat thickness measurement using optical technique with miniaturized chip leds: A preliminary human study," in 2007 29th Annual International Conference of the IEEE Engineering in Medicine and Biology Society, pp. 4548-4551, Aug 2007.

[13] A. Dupont, E. Sauerbrei, P. Fenton, P.Shragge, G. Loeb, and F. Richmond, "Real-time sonography to estimate muscle thickness: Comparison with mri and ct," Journal of Clinical Ultrasound, vol. 29, no. 4, pp. 230236.

[14] H. Horsman and A. Leach, "The estimation of the cross-sectional area of the ulna and radius," American Journal of Physical Anthropology, vol. 40 , no. 2, pp. 173-185.

[15] S. Gabriel, R. Lau, and C. Gabriel, "The dielectric properties of biological tissues: Iii. parametric models for the dielectric spectrum of tissues," Physics in Medicine \& Biology, vol. 41, no. 11, p. 2271, 1996.

[16] F. Ulaby, E. Michielssen, and U. Ravaioli, Fundamentals of Applied Electromagnetics. Prentice Hall, 6 ed., 2010.

[17] "International commission on non-ionizing radiation protection. guidelines for limiting exposure to time-varying electric, magnetic, and electromagnetic fields (up to $300 \mathrm{ghz}$ )," Health Physics, vol. 74, no. 4, pp. 494-522, 1998. 